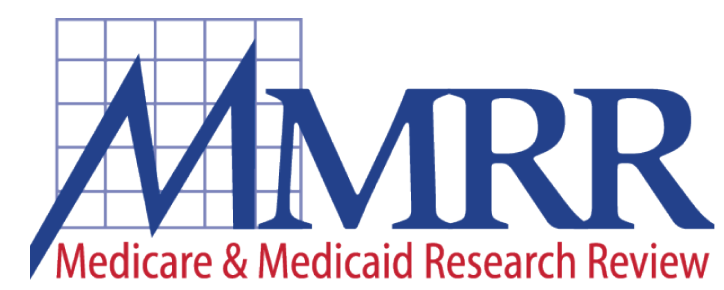

2013: Volume 3, Number 3

A publication of the Centers for Medicare \& Medicaid Services, Office of Information Products \& Data Analytics

\title{
Standardizing Medicare Payment Information to Support Examining Geographic Variation in Costs
}

\author{
Brian E. O’Donnell, ${ }^{1}$ Kathleen M. Schneider, ${ }^{2}$ John M. Brooks, ${ }^{3}$ Gregory Lessman, ${ }^{1}$ June Wilwert, ${ }^{1}$ \\ Elizabeth Cook, ${ }^{3}$ Glenda Martens, ${ }^{1}$ Kara Wright, ${ }^{4}$ Elizabeth A. Chrischilles ${ }^{4}$ \\ ${ }^{1}$ General Dynamics Information Technology \\ ${ }^{2}$ Schneider Research Associates, LLC \\ ${ }^{3}$ University of Iowa College of Pharmacy \\ ${ }^{4}$ University of Iowa College of Public Health
}

Objectives: Examination of efficiency in health care requires that cost information be normalized. Medicare payments include both geographic and policy-based facility type differentials (e.g., wage index and disproportionate share hospital), which can bias cost comparisons of hospitals and averages across geographic areas. Standardizing payment information to remove the area- and policy-based payment differentials should normalize much of the observed geographic variability in payments, allowing for a more accurate comparison of resource use between providers and across geographic regions. Use of standardized payments will ensure that observed payment variation is due to differences in practice patterns and service use, rather than Medicare payment differences over which the providers have no control. This paper describes a method for standardizing claim payments, and demonstrates the difference in actual versus standardized payments by geographic region.

Study Design and Methods: We used a nationwide cohort of Medicare patients hospitalized with an acute myocardial infarction (AMI) in 2007, then limited our study to those with Medicare Part A and Part B fee-for-service (FFS), and Part D coverage $(\mathrm{n}=143,123)$. Standardized payment amounts were calculated for each Part A and Part B claim; standardized and actual payments were summed for all services for each patient beginning with the index hospitalization through 12 months post discharge.

Principal Findings: Without standardization of payments, certain areas of the country are mischaracterized as either high or low healthcare resource-consuming areas. The difference between actual and standardized payments varies by care setting.

Conclusions: Standardized payment amounts should be calculated when comparing Medicare resource use across geographic areas.

Keywords: Medicare, Administrative Data Uses, Health Care Costs, Medical Geography

ISSN: 2159-0354

doi: http://dx.doi.org/10.5600/mmrr.003.03.a06 


\section{Medicare \& Medicaid Research Review} 2013: Volume 3, Number 3

\section{Mission Statement}

Medicare \& Medicaid Research Review is a peerreviewed, online journal reporting data and research that informs current and future directions of the Medicare, Medicaid, and Children's Health Insurance programs. The journal seeks to examine and evaluate health care coverage, quality and access to care for beneficiaries, and payment for health services.

\section{http://www.cms.gov/MMRR/}

\section{U.S. Department of Health \& Human Services Kathleen Sebelius Secretary}

\section{Centers for Medicare \& Medicaid Services Marilyn Tavenner Administrator}

Editor-in-Chief

David M. Bott, Ph.D.

The complete list of Editorial Staff and Editorial Board members may be found on the MMRR Web site (click link): $\underline{\text { MMRR Editorial Staff Page }}$

Contact: $\underline{\text { mmrr-editors@cms.hhs.gov }}$

Published by the Centers for Medicare \& Medicaid Services.

All material in the Medicare \& Medicaid Research Review is in the public domain and may be duplicated without permission. Citation to source is requested.

\section{Introduction}

There is a substantial body of research examining geographic variation in medical spending in the United States (Wennberg \& Cooper, 1996; O’Connor et al., 1999; Zuckerman, Waidmann, Berenson, \& Hadley, 2010; Alhassani, Chandra, \& Chernew, 2012). In general, areas with high spending for patients with myocardial infarction (MI) do not have lower 30-day mortality rates (Stukel et al., 2012). Major policy decisions are being made as a result of this observed variation that is, seemingly, not correlated with better outcomes. Variation in spending has been interpreted as evidence that some areas are providing efficient care and others are wasteful (Fisher et al., 2003). Payment reform strategies in the United States have pointed to geographic variation in spending to justify a plethora of cost-trimming policies (Baicker \& Chandra, 2009). Current efforts include a migration toward value-based purchasing of medical services, rather than traditional fee-for-service (FFS) payment (e.g., Medicare's Value-Based Purchasing programs for hospitals and physicians; CMS, n.d.a).

Medicare policy factors that vary geographically and by facility type can create variability in Medicare payments that mask the true relationship between geography and medical resource use intensity. Nonetheless, investigators commonly use the actual payment amounts from Medicare claims rather than standardizing the data to remove these payment differentials. Measurement error could be a reason for failure to correlate spending with outcomes, and it is crucial that bias in measurement be removed.

The purpose of this paper is to establish a valid and reliable measure of the medical resources used to care for patients with an acute MI, and to determine whether there are remaining geographic differences in resource use. 


\section{Background}

Medicare administrative claims data have often been used to illustrate geographic differences in cost and quality of care (Wennberg \& Cooper, 1996; Fisher et al., 2003). Few other nationwide data sources exist that can be used for this purpose. However, by design, the Medicare payments incorporate geographic variability and other facility type factors, which complicate the examination of geographic variation in the medical resource costs of patient care. Other investigators have identified the need to account for the structure of the Medicare payment system (Zukerman et al., 2010) to calculate normalized costs, to facilitate comparison of medical resource use.

Actual Medicare hospital payments are adjusted: 1) for the acuity of care provided to the patient-there may be outlier payments for particular patients, and/or additional payments for use of new technology; 2) for the type of facilitysuch as whether the hospital is a critical access hospital(CAH), whether it serves a disproportionate share of Medicaid or uninsured patients (DSH), and/or whether it is a teaching hospital; and 3) for the location of the facility-which is generally reflected in the wage-index for the area. To create standardized payments for measuring resource use, it is appropriate to keep payment factors related to acuity of care, since these factors indicate intensity of resource use. However, a standardized payment amount should adjust for the latter two factors.

Zuckerman and colleagues adjusted Medicare claims payments by accounting for geographic variation in the Medicare provider wage index alone (Zuckerman et al., 2010); however, a more complete payment standardization, which also adjusts for the payment differentials for various care settings and service categories, is desirable to account for the full set of factors structured into the Medicare payment system. The Medicare Payment
Advisory Commission (MedPAC) was privy to the detailed Medicare fee schedule and payment factors in their role as an independent US Congressional agency. They engaged in this type of elaborate payment standardization process, and found substantial reduction in geographic variation in Medicare payments after standardization (Medicare Payment Advisory Commission, 2009). A thorough standardization of Medicare claims payments has not previously been accomplished by researchers outside of CMS or MedPAC. It is worthwhile to identify the necessary components of payment standardization for Medicare claims, to demonstrate how to remove these components from payment calculations to produce standardized payments for each major care setting, and to ascertain the benefits of engaging in this type of analysis in terms of understanding variation in medical resource use.

The objective of payment standardization is to remove the geographic and facility-specific variation in payment that is structured into the Medicare payment system, while leaving intact the variability in payments due to the volume and mix of services. The standardized payments allow for comparison of Medicare FFS medical resource utilization nationwide.

For the purposes of this study, we attempted to estimate a consistent measure of the medical resource costs used to care for patients, by adjusting payments for Medicare beneficiaries with a common occurrence-hospitalization for myocardial infarction (MI). Focusing on a single condition ensured that the resulting variation in our measure would not vary due to case-mix. MI is a very common and costly condition (Nichols, Bell, Pedula, \& O'Keefe-Rosetti, 2010), and the initial treatment of acute MI is not considered discretionary or supply-sensitive care; it is urgent care. There is fairly widespread agreement regarding necessary components of $\mathrm{MI}$ initial patient evaluation and treatment (Krumholz et al., 2008). Furthermore, there is evidence ofgeographic variation 
in terms of service use and medical spending for MI (O'Connor et al., 1999; Lucas, Sirovich, Gallagher, Siewers, \& Wennberg, 2010).

The current study extends previous research by providing a rationale and context for calculating standardized Medicare payments. We demonstrate geographic variation in medical resource use with cost metrics that have completely removed the geographic and facility-type payment differentials due to Medicare policy, and evaluate the impact of payment standardization by comparing actual and standardized payments across geographic areas for patients treated for a particular acute condition. This study also provides a framework for greater transparency and consistency in measuring medical resource use, so that future studies relying on information regarding payment variation will be better informed.

\section{Methods}

\section{Population}

Our cohort included Medicare beneficiaries aged 65 and older, hospitalized for an acute MI in 2007, who were enrolled in Medicare Part A and B feefor-service (FFS) rather than managed care (known as Medicare Advantage) for the year after discharge, or until time of death, and enrolled in Medicare Part D prescription drug coverage for at least one month of 2007. The final sample size was 143,123.

\section{Data sources}

We extracted Chronic Condition Warehouse (CCW) data files for all Medicare enrollment and A/B FFS claims for this population from (2007 and 2008). We calculated all Medicare FFS payments for the index hospitalization and up to one year following the index hospital discharge date, using all Medicare institutional and non-institutional (both Medicare Part A and B) claims. We obtained the Hospital Referral Regions' (HRR) categories from the Dartmouth Atlas (Dartmouth Atlas, n.d.). We used their ZIP code crosswalk file from 2007 to classify the HRR for each person during 2007.

We gathered information regarding specific payment amounts from a variety of sources:

1. Base payment rates from the Federal Register (see, for example, Federal Register, 2011);

2. Diagnosis related group (DRG) weights (see, for example, Table 5 in CMS, n.d.b);

3. Fee schedules, such as the physician fee schedule, from the CMS Web site (CMS, n.d.c); and

4. Wage index information from the CMS Web site (CMS, n.d.d).

One of the authors (B. E. O.) had extensive interactions with CMS through a separate contract tocalculatestandardized Medicare payments, which allowed for the opportunity to obtain additional CMS payment schedules and cross-walks that were not immediately available online. The inventory of the information needed to geographically standardize payments in all Medicare institutional and non-institutional settings is documented in a methodology paper posted by CMS on the QualityNet Web site (CMS, n.d.e), and is also included as supplemental content.

\section{Costing Methods}

We used all Medicare Part A institutional (i.e., inpatient [IP], skilled nursing facility [SNF], hospice [HOS], and home health $[\mathrm{HH}])$, and Part B institutional (i.e., hospital outpatient [HOP]), claims where the actual payment amount (CLM_ PMT_AMT) was greater than or equal to $\$ 0$. For Part B non-institutional claims (i.e., physician/ carrier and durable medical equipment [DME]), we kept all line items for the claim where the line processing indicator showed the service was allowed under Medicare (i.e., values A, R, or S). 
Throughout this paper, we use the terms total payments to mean the total provider payments for a service-including the insurance (Medicare) and patient portion of the payment (which may include deductible and coinsurance amounts). If Medicare is not the primary payer, then any dollar amount paid by a third party is not factored into either the actual or standardized payment (i.e., in accordance with CMS methodology, other payer amounts are ignored). We first calculated the total payments for all people in our sample using the actual provider payments as they were documented on the Part A and B claims, including the Medicare payment and beneficiary payment amounts. For institutional claims, this included outlier payments, when applicable. All payments for patients in the MI sample were included for the acute hospitalization through one year post care, regardless of the reason for care.

\section{Standardization Methods}

In addition to the actual provider payments, we calculated standardized provider payments. The payment system for Medicare is complex, with a combination of factors determining the ultimate provider/facility reimbursement from Medicare. The structure and components of payment vary by care setting and provider type. Since our objective was to identify and remove payment differentials related to geography and policy-related factors, we began the standardization process by preserving all information on the claim related to the acuity of care and different types of services provided. Exhibit 1

\section{Exhibit 1. Factors Influencing Medicare Provider Payment Rates, by Setting}

Payment components which indicate resource use Factors to standardize

A. Institutional (i.e., IP, SNF, HH, and HOS claims)-Medicare Part A:

1. The base payment rate for the service

2. A DRG weight for inpatient claims, a Resource Utilization Group (RUG) weight for SNF claims and a HH Resource Group (HHRG) weight for HH claims

3. Additional payments to account for patient/care factors-outlier payment, short stay adjustments, etc.

B. Institutional (i.e., HOP claims)-Medicare Part B:

1. Fee-schedules, primarily: Ambulatory payment classifications (APC) fee schedule Medicare Physician Fee Schedule (MPFS) for physician services

Lab fee schedule for lab services
1. The wage index for the provider/facility area

2. Additional payments to account for facility factors-teaching hospitals (graduate medical education [GME], indirect medical education [IME], hospitals serving a disproportionate share (DSH) of Medicaid or uninsured patients

C. Non-Institutional (i.e., Physician/supplier and DME claims)-Medicare Part B:

1. Fee-schedules, which include the conversion factors (similar to a base rate) and relative value units (RVU) to adjust for acuity and care setting. For example:

MPFS for physician services

Ambulatory surgery center (ASC) schedule

Anesthesia schedule

Lab fee schedule

DME fee schedule
1. Geographic practice cost index for the provider/facility area 
identifies the major components of Medicare payments, and indicates which of the payment factors were removed in the standardization process.

There are more Medicare payment factors that are standardized for Part A as compared to Part B. We examined the impact of standardization on both claim types, and also examined the differences between actual and standardized payments for a variety of settings.

Although the actual Medicare payments appear on the claims, it is instructive to understand the Medicare payment factors that are applied to various care settings and service types. Typically, there are one or more base payment rates that apply for a particular setting. The base rates are multiplied by factors that adjust payment for the specific service rendered and the wage index of the service location. Other facility or procedure specific payment adjustments, including add-on payments or outlier payments, may then be added. The payment standardization process recreates the formula used to compute the actual payment, but omits the geographic and facility specific adjustments. The computations are illustrated with a couple of examples.

\section{Illustrative Example 1-Inpatient Claims}

The general formula for actual Medicare acute inpatient hospital payments (i.e., for those paid using the Medicare inpatient prospective payment system [IPPS], which are billed as Part A services) is:

Actual IPPS payment $=\left[\right.$ operating base ${ }^{*}$ labor share ${ }^{\star}$ wage index + operating base ${ }^{\star}(1$-labor share $)+$ capital base $^{*}$ (wage index) $)^{\text {capital adjustment })]^{*}}$

DRG weight + operating IME + capital IME + operating $\mathrm{DSH}+$ capital DSH + add-on payments + (nch_drg_outlier_aprvd_pmt_amt + clm pps_cptl_outlier_amt)
If one were to compute the actual Medicare payment, the labor portion of the operating base and capital base are adjusted for area-based cost of living using the wage index. Then the sum of the adjusted labor base, the unadjusted non-labor base and the adjusted capital base is multiplied by the DRG weight. The operating and capital indirect medical education (IME) and disproportionate share hospital (DSH) payments are added. Finally, the outlier and/or add-on payments are added.

To compute the standardized payment, the formula for computing the actual payment is changed as follows: the labor base and capital base are not adjusted to account for area-based wage indexes, and the IME and DSH payments are not included. The formula is simplified, and becomes:

$$
\begin{aligned}
& \text { Standardized IPPS payment }=[\text { operating } \\
& \text { base }+ \text { capital base }]^{*} \text { DRG weight }+ \text { add-on } \\
& \text { payments }+ \text { standardized outlier payments }{ }^{1}
\end{aligned}
$$

An example is given for two inpatient prospective payment system (IPPS; i.e., acute hospital) heart failure claims (DRG 127). The same service was performed by two Delaware (DE) hospitals in different wage index areas. These providers have different levels of payments for IME and DSH due to their facility characteristics; for simplicity, no outlier or add-on payments were included. The parameter values and calculation results are shown below. The standardized payment for the service is the same for both hospitals, but the actual Medicare payments were different due to differences in wage index and facility payments for IME and DSH (Exhibit 2).

\footnotetext{
${ }^{1}$ Outlier payments are geographically standardized using the following formula: nch_drg_outlier_aprvd_pmt_amt/(wage indx*labor share.+non-labor share) + clm_pps_cptl_outlier_amt/ (wage_ind $x^{* *}$ capital adjustment factor)
} 
Exhibit 2. Example of Inpatient Payment Standardization for 2007

\begin{tabular}{lcc}
\hline DRG 127 & DE Provider 1 & DE Provider 2 \\
\hline Labor_Base & $\$ 3,397.52$ & $\$ 3,397.52$ \\
Nonlabor_Base & $\$ 1,476.97$ & $\$ 1,476.97$ \\
Capital_Base & $\$ 427.03$ & $\$ 427.03$ \\
DRG_Weight & 1.049 & 1.049 \\
Capital_Adjustment & 0.6848 & 0.6848 \\
Wage_Index & 1.0684 & 1.0088 \\
Operating IME & $\$ 602.23$ & $\$ 446.34$ \\
Operating DSH & $\$ 204.27$ & $\$ 619.36$ \\
Capital IME & $\$ 19.05$ & $\$ 45.49$ \\
Capital DSH & $\$ 25.97$ & $\$ 22.47$ \\
Actual Payment & $\mathbf{\$ 6 , 6 7 7 . 3 5}$ & $\mathbf{6 , 7 2 9 . 0 1}$ \\
Standardized Payment & $\mathbf{\$ 5 , 5 6 1 . 2 9}$ & $\mathbf{\$ 5 , 5 6 1 . 2 9}$ \\
\hline
\end{tabular}

SOURCE: Calculations based on CCW Medicare Part A fee-for-service claims, 2007-2008, and other data sources.

\section{Illustrative Example 2-Physician Office Claims}

The base payment rate for Part B non-institutional services requires use of fee schedules, such as the Medicare Physician Fee Schedule (MPFS) for physician services. The MPFS includes: the work relative value units (RVU), practice expense (PE) RVU, and malpractice (MP) RVU adjusted by their respective geographic practice cost indices (GPCI). This GPCI inserts area-based differentials into the payment formula. Then the sum of the adjusted rates is multiplied by the payment conversion factor. A work adjustment factor is also included, which was only used in 2007 and 2008 to achieve CMS "budget neutrality." The formula for actual Medicare payments for a provider using the Medicare Physician Fee Schedule (MPFS) is:

\section{Actual MPFS payment $=[$ WORK RVU * WORK GPCI ${ }^{\star}$ WORK Adjustment Factor + PE RVU * PE GPCI + MP RVU * MP GPCI ] ${ }^{\star}$ Conversion Factor}

To compute the standardized payment, the RVUs are not adjusted geographically; therefore, the GPCI is not used. The formula becomes:

\section{Standardized MPFS payment $=[$ WORK $\mathrm{RVU}^{\star}$ WORK Adjustment Factor $+\mathrm{PE}$ RVU + MP RVU ] * Conversion Factor}

To illustrate the differences in the actual versus the standardized payment factors, another example is presented. This example describes the payment calculations for visits to two physician offices using the Medicare Physician Fee Schedule (MPFS) including the conversion factor and several RVUs. The same service was performed by two different providers, one in Iowa (IA) and one in New Hampshire (NH).

The two providers submitted claims for payment in 2007 that had a CMS/HCFA Common Procedure Coding System (HCPCS) code 99213 on the claims. This code indicates the patient was seen for an office or outpatient visit, the patient was an established patient, and the visit was an expanded problem-focused visit (e.g., for managing a patient who had recently been hospitalized). The parameter values and calculation results are shown below where, again, the effect of payment standardization is apparent (see Exhibit 3).

These are common examples of payment calculations for Part A and Part B services, but the 
Exhibit 3. Example of Office/Outpatient Visit, HCPCS 99213, in 2007

\begin{tabular}{llcc}
\hline Payment Component & \multicolumn{1}{c}{ HCPCS 99213 } & IA Provider & NH Provider \\
\hline MPFS & Conversion Factor (e.g., base amount & & \\
& for the HCPCS) & 37.8975 & 37.8975 \\
& Work RVU & 0.92 & 0.92 \\
& PE RVU & 0.71 & 0.71 \\
& MP RVU & 0.03 & 0.03 \\
& Work Adjustment & 0.8994 & 0.8994 \\
Geographic adjusters & Work GPCI & 1 & 1 \\
& PE GPCI & 0.869 & 1.029 \\
& MP GPCI & 0.579 & 0.927 \\
Actual Payment & & $\mathbf{\$ 5 5 . 4 0}$ & $\mathbf{\$ 6 0 . 1 0}$ \\
Standardized Payment & & $\mathbf{\$ 5 9 . 4 0}$ & $\mathbf{\$ 5 9 . 4 0}$ \\
\hline
\end{tabular}

SOURCE: Calculations based on CCW Medicare Part B fee-for-service claims, 2007-2008, and other data sources.

formulas differ a great deal by care setting. Also, there are many special situations that require adjustments to the general formulas for payment calculations (e.g., short stay hospital claims; Maryland IPPS claims, which CMS handles differently due to a waiver; physician claims with multiple services; certain types of imaging claims, etc.).

The Medicare base rates and adjustment factors are revised annually. Some care settings use a calendar year cycle for periodic adjustments, whereas others use a fiscal year cycle and some use a rate year cycle. We have not performed any additional adjustment to reflect either 2007 or 2008 dollars, although our observation period straddles both years for all patients; the index hospitalization was initiated in 2007 and a full year of post-hospitalization observation was obtained. For this paper, where our objective is to highlight differences between an actual and standardized payment method, we feared that additional adjustments may introduce an added layer of complexity that is undesirable at this time. Our estimates will only be affected if the distribution of index MIs varies within HRRs across time.

The objective of the payment standardization is to remove the geographic and facility-specific variation in payment that is structured into the Medicare payment system, while leaving intact the variability in payments due to the volume and mix of services. The geographically standardized payments allow for comparison of Medicare FFS medical resource utilization nationwide.

\section{Statistical Analysis}

Two different perspectives on total Medicare payments (actual versus standardized) were contrasted by initially calculating total provider payments, beginning at the index hospital admission through a period of one year after discharge from the acute hospitalization.

Standardization is applied at the claim or line item level. Total actual and standardized payments were calculated using HRRs as the primary geographic unit of analysis. The HRR was assigned to each beneficiary using the beneficiary ZIP code in the Medicare enrollment data, which is the mailing address for Medicare billing correspondence. We also examined payments for various care settings. For each HRR, per member per month (PMPM) payments were calculated.

The geographic variability in payments was examined by observing the HRRs with the highest 
and lowest payments (actual and standardized). HRR maps were generated to display the overall variation in actual payments and standard payments. The difference in the variation and central tendency of the distributions of actual and standard payments was examined. The HRRs were ranked by quintile for actual payments and standard payments, and the distribution of the differences in quintile ranking is displayed to show the effect standardization has when attempting to compare HRRs.

Total actual and standardized payments were calculated for specific settings (i.e., inpatient services, home health services, etc.). We used box plots to illustrate the difference in the distributions of actual and standard payments for each of the institutional care settings.

\section{Results}

A total of 143,123 Medicare FFS beneficiaries with MI met the study inclusion criteria. Standardizing Medicare provider payments resulted in lower payment amounts overall, compared to the actual payments. For inpatient claims with a large difference between the actual and standardized payment, this is largely due to removal of IME and DSH payments.

The unadjusted national Medicare payments for all services for AMI patients in the sample from the index admission date through one year post-discharge were $\$ 7,072,269,216$; the standardized payments were $\$ 6,511,846,903$. The index hospitalization accounted for $38.7 \%$ of actual payments during this surveillance period and $36.8 \%$ of standardized payments.

\section{Exhibit 4. Mean Per Capita Spending for Index MI Through One Year Per HRR for Actual and Standardized} Payments

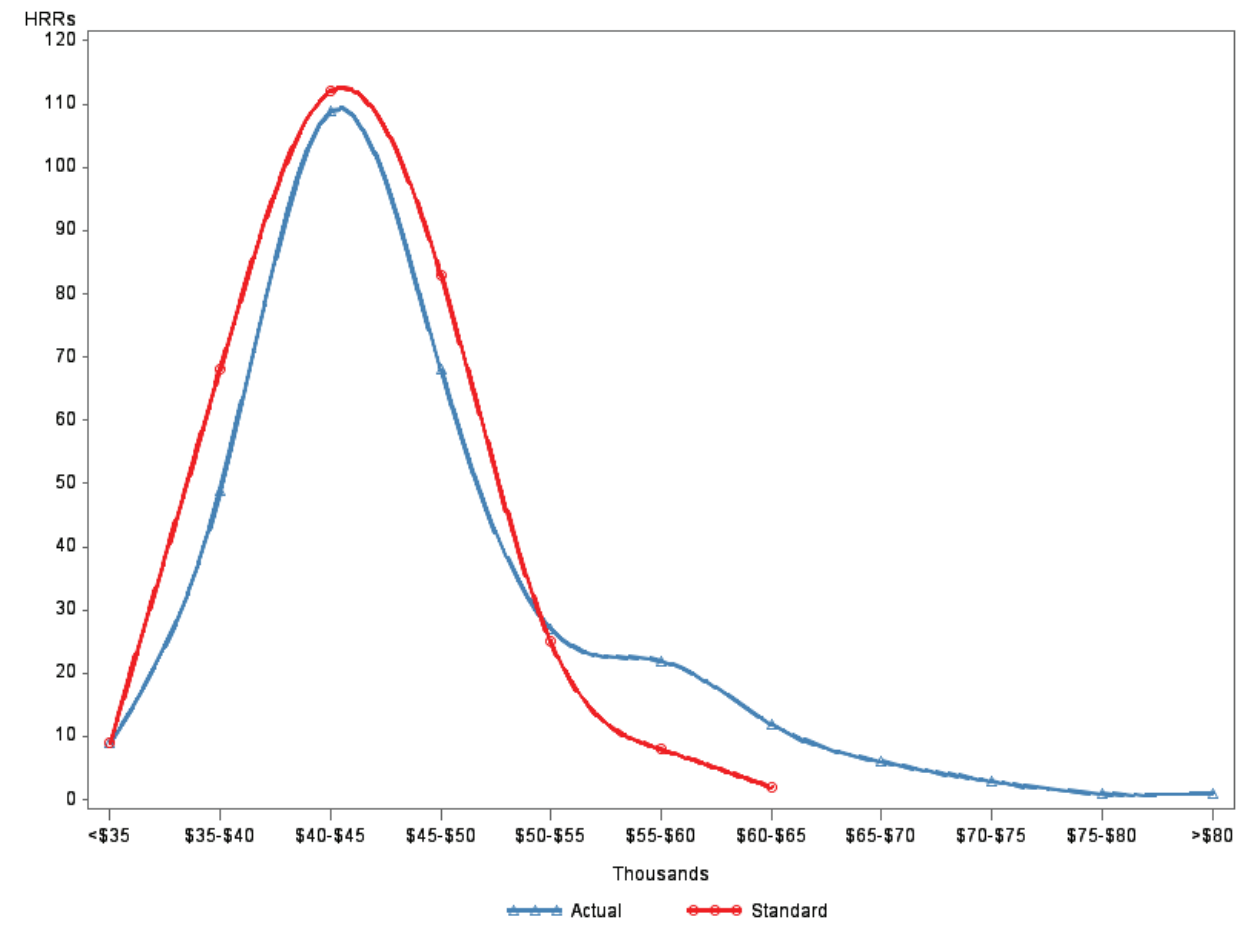

SOURCE: Calculations based on CCW Medicare enrollment and Part A and B fee-for-service claims, 2007-2008,

Dartmouth Atlas HRRs 2007, and other data sources. 
The average (mean) payments for all patients within an HRR were calculated for the index MI through one year post. The distribution of these HRR-average payments is displayed for both the actual and geographically standardized payments in Exhibit 4 ( $\mathrm{n}=306$ HRRs).

The average HRR actual Medicare payment for the index through one year was $\$ 46,416$ (median = $\$ 44,478)$. The average HRR standardized payment was $\$ 43,703$ (median $=\$ 42,824$ ). The standardized payments were more normally distributed than the actual payments. The net impact of standardizing payments substantially reduced overall variation between HRRs in terms of average per capita payment, and reductions in the mean and median payment amounts.

Different HRRs emerge as having high or low per member per month (PMPM) payments when using actual, as compared to standardized, payment information. The 10 highest and lowest HRRs are displayed in Exhibit 5, along with the average PMPM provider payments for the observation period.

Areas of New York are not ranked among the 10 highest HRRs after standardization, while areas in Louisiana (LA) are identified as highspending areas after standardization. Similarly, areas of Kentucky (KY) are ranked among the

Exhibit 5. Ten Highest and Lowest HRRs, in Terms of PMPM, for Actual and Standardized Payments

\begin{tabular}{|c|c|c|c|}
\hline \multicolumn{4}{|c|}{10 Highest HRRs } \\
\hline \multicolumn{2}{|c|}{ Actual PMPM \$ } & \multicolumn{2}{|c|}{ Standardized PMPM \$ } \\
\hline NY-Bronx & $\$ 9,638.62$ & FL-Miami & $\$ 7,518.42$ \\
\hline NY-Manhattan & $\$ 9,366.84$ & TX-McAllen & $\$ 7,061.13$ \\
\hline NJ-Newark & $\$ 8,710.50$ & TX-Harlingen & $\$ 6,857.73$ \\
\hline FL-Miami & $\$ 8,630.27$ & MI-Dearborn & $\$ 6,794.02$ \\
\hline CA-Los Angeles & $\$ 8,268.99$ & NJ-Newark & $\$ 6,657.88$ \\
\hline IL-Chicago & $\$ 7,911.02$ & IL-Chicago & $\$ 6,592.19$ \\
\hline TX-McAllen & $\$ 7,802.97$ & LA-Monroe & $\$ 6,510.06$ \\
\hline CA-Contra Costa County & $\$ 7,760.87$ & CA-Los Angeles & $\$ 6,499.02$ \\
\hline NY-East Long Island & $\$ 7,753.67$ & LA-Baton Rouge & $\$ 6,407.84$ \\
\hline TX-Harlingen & $\$ 7,698.02$ & LA-New Orleans & $\$ 6,388.22$ \\
\hline \multicolumn{4}{|c|}{10 Lowest HRRs } \\
\hline \multicolumn{2}{|c|}{ Actual PMPM \$ } & \multicolumn{2}{|c|}{ Standardized PMPM \$ } \\
\hline ND-Bismarck & $\$ 3,484.62$ & CO-Grand Junction & $\$ 3,608.43$ \\
\hline ID-Idaho Falls & $\$ 3,552.26$ & ID-Idaho Falls & $\$ 3,631.69$ \\
\hline IA-Sioux City & $\$ 3,654.73$ & ND-Bismarck & $\$ 3,747.12$ \\
\hline IA-Waterloo & $\$ 3,680.91$ & NY-Binghamton & $\$ 3,759.45$ \\
\hline MT-Missoula & $\$ 3,683.78$ & IA-Sioux City & $\$ 3,760.77$ \\
\hline IA-Dubuque & $\$ 3,766.63$ & IA-Waterloo & $\$ 3,775.34$ \\
\hline NY-Binghamton & $\$ 3,798.76$ & MT-Missoula & $\$ 3,804.36$ \\
\hline CO-Grand Junction & $\$ 3,798.84$ & OR-Medford & $\$ 3,807.52$ \\
\hline IA-Cedar Rapids & $\$ 3,803.19$ & OR-Bend & $\$ 3,829.57$ \\
\hline KY-Paducah & $\$ 3,978.64$ & OR-Eugene & $\$ 3,830.38$ \\
\hline
\end{tabular}

SOURCE: Calculations based on CCW Medicare enrollment and Part A and B fee-for-service claims, 2007-2008, Dartmouth Atlas HRRs 2007, and other data sources. 
Exhibit 6. Distribution of HRR Total Actual, Followed by Total Standardized, Medicare Provider Payments for treatment of MI Patients.
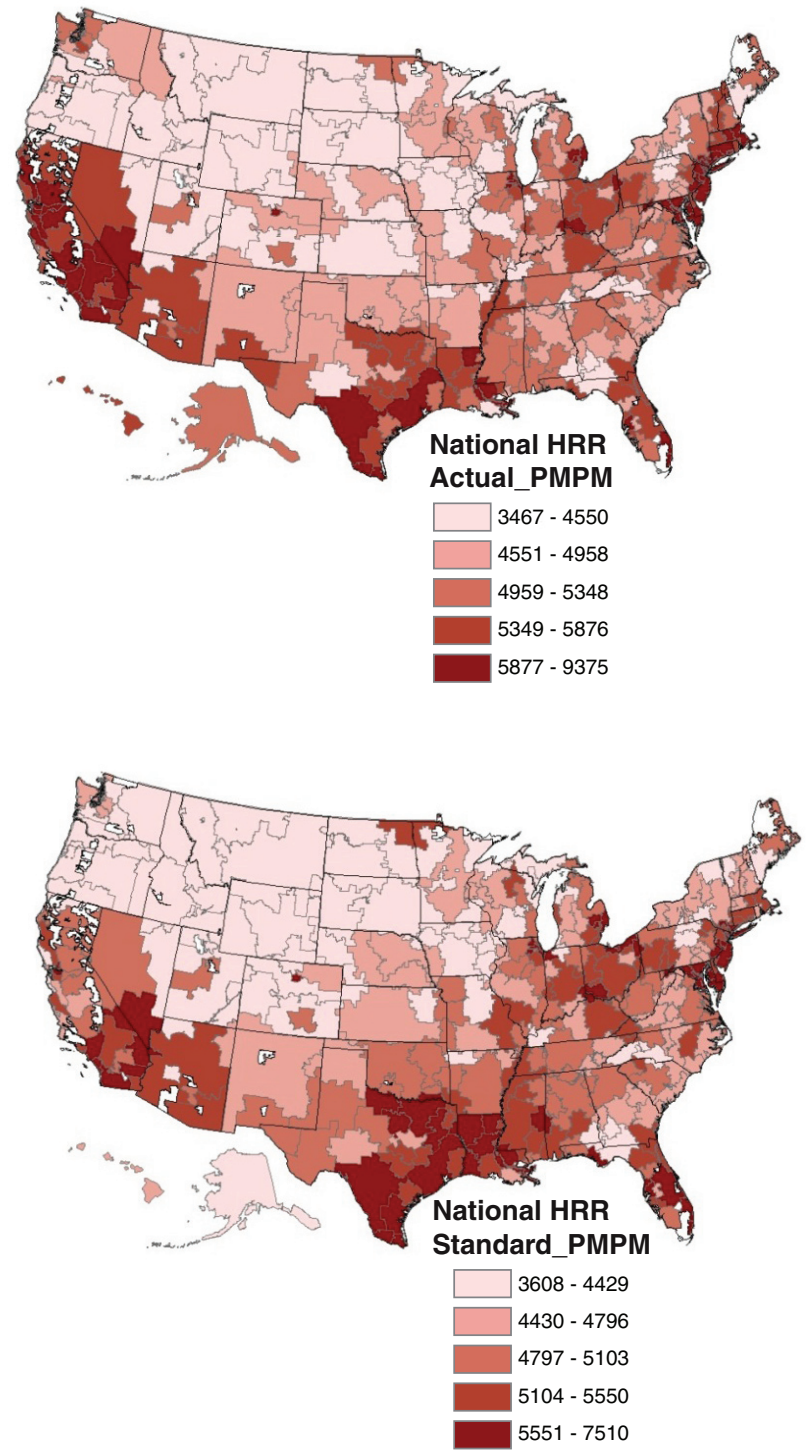

SOURCE: Calculations based on CCW Medicare enrollment and Part A and B fee-for-service claims, 2007-2008, Dartmouth Atlas HRRs 2007, and other data sources.

lowest spending areas prior to standardization, yet after standardization they are not in the 10 lowest HRRs; areas in Oregon (OR) appear to be providing low-cost care once standardized payment information is used.

The nationwide distribution of total provider payments for the observation period is mapped, by HRR, two different ways. The first map displays HRR-level actual provider payments and the second map uses the standardized provider payments (Exhibit 6). Although some of the same HRRs are in the highest quintile regardless of whether the actual or standardized payments are used (e.g., Miami, FL and McAllen, TX), other HRRs that appeared high using actual payments shifted to lower quintiles after payments were standardized (e.g., Contra Costa County, CA; Bronx and Manhattan, NY). 
In order to determine the ranking of high and low HRRs, the actual and standardized payments were grouped into quintiles and the difference in quintile ranking based on actual payments and standardized payments was computed (Exhibit 7).

Exhibit 7. Quintile Changes ${ }^{1}$ in HRRs, When Comparing Actual to Standardized Payments $\left(n=307^{2}\right)$

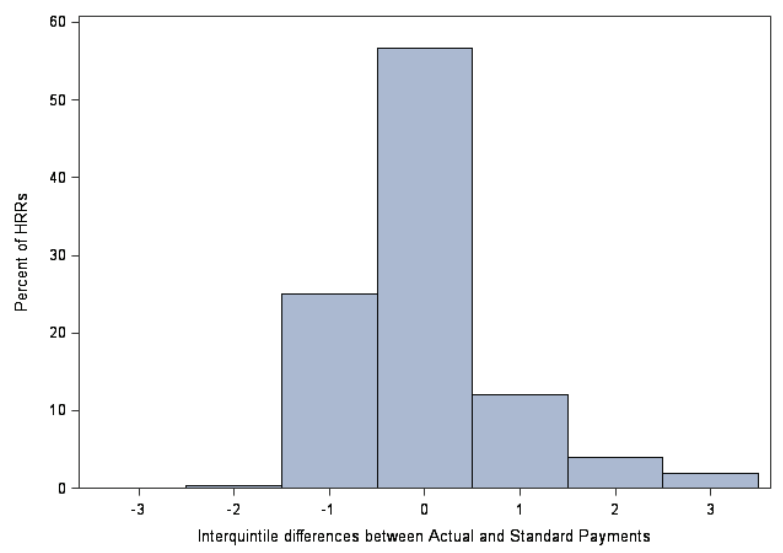

${ }^{1}$ Negative numbers indicate the standardized payments for the HRR were higher than the actual payments.

${ }^{2} 306$ HRRs are used; there is also an "unknown" HRR category used when a beneficiary ZIP code could not be assigned to an HRR. SOURCE: Calculations based on CCW Medicare enrollment and Part A and B fee-for-service claims, 2007-2008, Dartmouth Atlas HRRs 2007, and other data sources.
Almost half the HRRs change quintile ranking when standard payments are used instead of actual payments. For $2 \%$ of the HRRs (6 HRRs), the ranking drops three quintiles when the standardized payment is used to rank the HRR instead of the actual payment. For 19 of the 306 HRRs (6\%), the ranking shifts two or more quintiles after standardization.

\section{Payment Standardization Impact by Care Setting}

The impact of payment standardization varies by care setting. The standardization algorithms apply different adjustment factors to different care settings. When comparing actual to standardized payments, the net effect was that standardization resulted in lower payments for inpatient care, but higher estimates for all other settings except for hospice (Exhibit 8).

The magnitude of the difference between actual and standardized payments is very large for this population using IP care (14.4\% of the actual payment), yet quite small for settings such as hospice ( $0.53 \%$ of actual payments). Also, PMPM differences in actual and standardized payments

Exhibit 8. Setting-Specific Total and PMPM Payments Using Both Actual and Standardized Payments

\begin{tabular}{|c|c|c|c|c|c|c|c|}
\hline \multirow[b]{2}{*}{ Setting } & \multicolumn{2}{|l|}{ Actual } & \multicolumn{2}{|c|}{ Standardized } & \multicolumn{3}{|c|}{ Difference } \\
\hline & Total Payments & PMPM & Total Payments & PMPM & $\begin{array}{c}\text { Payment } \\
\text { Difference }\end{array}$ & $\begin{array}{c}\text { PMPM } \\
\text { Difference }\end{array}$ & $\begin{array}{c}\% \\
\text { Change }\end{array}$ \\
\hline \multicolumn{8}{|c|}{ Institutional Part A } \\
\hline IP & $\$ 4,224,071,357.43$ & $\$ 3,368.26$ & $\$ 3,617,279,899.81$ & $\$ 2,884.40$ & $\$ 606,791,457.62$ & $\$ 483.85$ & $14.37 \%$ \\
\hline SNF & $\$ 768,795,645.54$ & $\$ 1,878.70$ & $\$ 790,291,493.13$ & $\$ 1,931.23$ & $(\$ 21,495,847.59)$ & $(\$ 52.53)$ & $-2.80 \%$ \\
\hline HOS & $\$ 141,645,320.18$ & $\$ 1,381.03$ & $\$ 140,898,448.27$ & $\$ 1,373.75$ & $\$ 746,871.91$ & $\$ 7.28$ & $0.53 \%$ \\
\hline $\mathrm{HH}$ & $\$ 250,762,719.41$ & $\$ 495.55$ & $\$ 259,875,143.29$ & $\$ 513.56$ & $(\$ 9,112,423.88)$ & $(\$ 18.01)$ & $-3.63 \%$ \\
\hline \multicolumn{8}{|c|}{ Institutional Part B } \\
\hline $\mathrm{HOP}$ & $\$ 433,099,076.96$ & $\$ 388.84$ & $\$ 443,050,955.45$ & $\$ 397.77$ & $(\$ 9,951,878.49)$ & $(\$ 8.93)$ & $-2.30 \%$ \\
\hline \multicolumn{8}{|c|}{ Non-Institutional Part $\mathbf{B}^{1}$} \\
\hline PHYS & $\$ 863,702,333.86$ & $\$ 689.25$ & $\$ 868,497,398.97$ & $\$ 693.08$ & $(\$ 4,795,065.11)$ & $(\$ 3.83)$ & $-0.56 \%$ \\
\hline OPTB & $\$ 390,192,762.94$ & $\$ 312.30$ & $\$ 391,953,563.62$ & $\$ 313.71$ & $(\$ 1,760,800.68)$ & $(\$ 1.41)$ & $-0.45 \%$ \\
\hline
\end{tabular}


are very small for the non-institutional settings $(0.56 \%$ of actual payments for physician office care and $0.45 \%$ for other Part B services).

We created a display of the distribution of average HRR-level PMPM payments using box plots to illustrate the impact of performing payment standardization with data files for the various care settings ( $\mathrm{n}=306$ HRRs). For each institutional care setting, the box plot compares the variability in standardized and actual provider payments (Exhibit 9).

For IP care, there is a very large impact on payment variability after payments are standardized. The mean HRR-level PMPM payments are much lower when standardized, and the variability in payments is compressed.
For SNFs, standardizing the payments slightly increases the average payment, and has little effect on the variability of payments. For HOS, the average payments are nearly identical after standardization; however, variability in HRR-level PMPM payments is slightly reduced. Very little impact on HRR-level PMPM payment is seen for the other non-institutional care settings and the HOP setting (data not shown).

\section{Discussion}

Standardizing Medicare payment information, to remove the area-and policy-based payment differentials, is important if the objective is to

Exhibit 9. HRR-level Distribution of Average PMPM Actual and Standardized Provider Payments for Institutional Settings

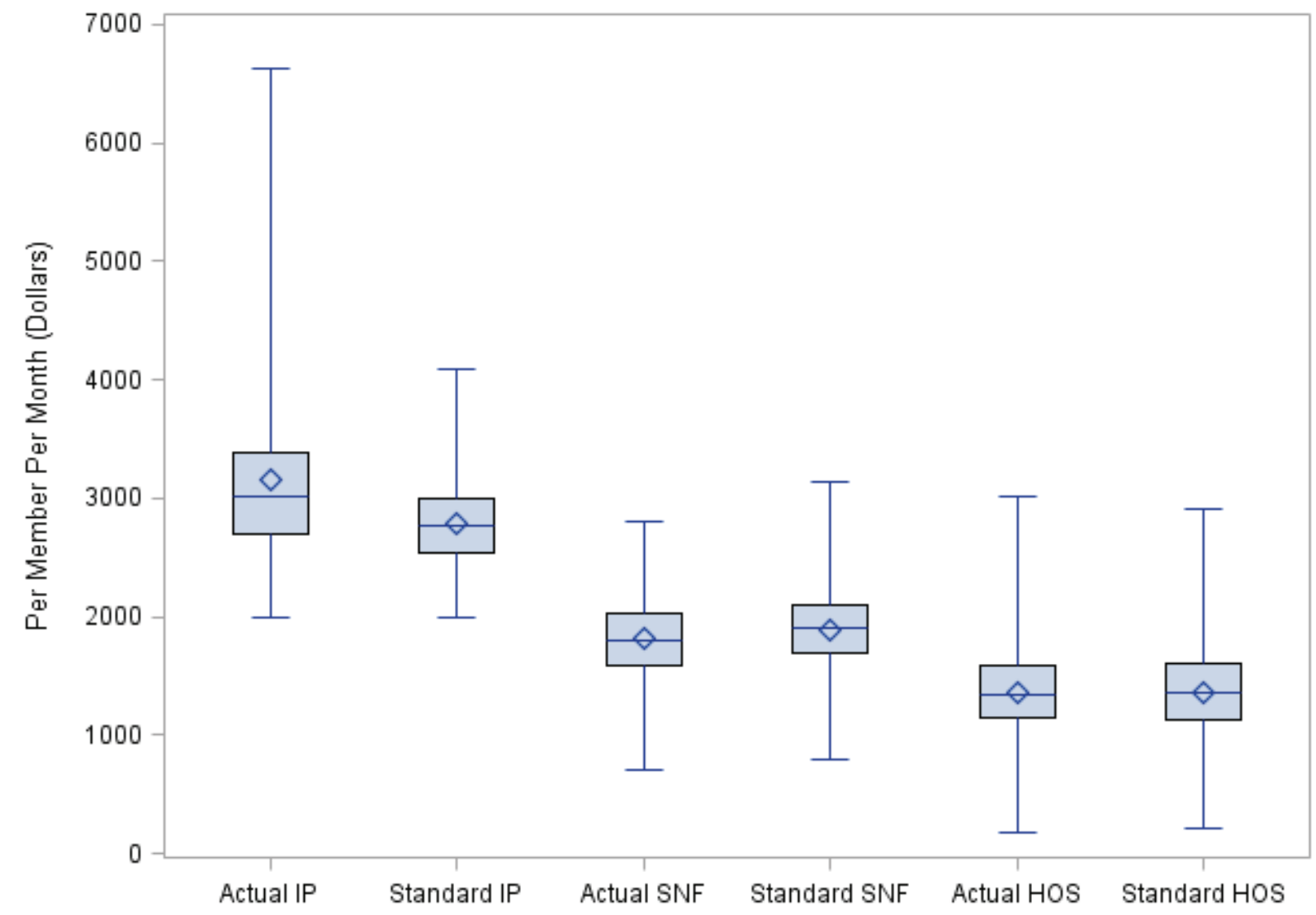

SOURCE: Calculations based on CCW Medicare enrollment and Part A and B fee-for-service claims, 2007-2008, Dartmouth Atlas HRRs 2007, and other data sources. 
compare resource use between providers and across geographic regions. In the actual payment map (Exhibit 6), it appears that California is one of the highest cost regions (in the upper quintile). However, in the standardized payment map, it is apparent that California is not that costly, instead Louisiana (e.g., Monroe, Baton Rouge, and New Orleans) and Florida (e.g., Panama City) are among the most costly regions. Examination of the HRR-level payments demonstrated that different areas of the country are identified as high or lowspending areas, depending on whether actual or standardized payments were used.

If a researcher wants to compare HRRs in terms of average payments or efficiency, then standardization can have an important effect. In fact, 19 of the 306 HRRs (6\%) shifted two or more quintiles in payment ranking after standardization (Exhibit 7). Using actual payment data to test hypotheses about which regions of the country have particularly high or low spending can lead to false conclusions. For example, the maps in Exhibit 6 show that actual PMPM spending in most of Louisiana is not in the top quintile, but almost all of Louisiana is in the top quintile for standardized PMPM spending. In fact, Exhibit 5 shows that no HRR in Louisiana is in the top 10 in actual PMPM spending, but three Louisiana HRRs are in the top 10 in standardized PMPM spending. Similarly, Exhibit 5 shows that using actual PMPM spending in Oregon does not appear to have particularly low PMPM spending, but three Oregon HRRs are in the bottom 10 in standardized PMPM spending.

Using actual Medicare payments would automatically classify all high cost geographic areas (e.g., areas with high labor costs) as using relatively more resources to care for patients than areas with lower labor costs (Exhibit 5). This bias would be particularly important to address if payment incentive programs were to reward efficiency in caring for patients. Understanding the appropriate configuration of care to most efficiently deliver quality health care requires careful identification of the most and least efficient areas and providers, which is difficult given the limitations of current Medicare claims data. Incorrect attributions regarding which areas of the country provide highor low-cost care are likely using actual, rather than standardized, Medicare payment amounts.

The net impact of standardizing payment data was to reduce both the mean and median per capita payments, and to substantially reduce the overall variation between geographic areas (Exhibit 4). For this population, all of whom required hospital care at least for the initial MI treatment, the care setting where the impact of payment standardization was the largest was IP care. This large difference in payments is likely due to two factors: (1) The Medicare payment policy requiring both geographic and facility differentials for actual IP payments, and (2) All members of this population had at least one hospitalization due to the design of the study. It is possible, if one had a population without substantial hospital use, or perhaps one with extensive use of ambulatory care for disease management, that a somewhat lesser effect of standardization may be observed in inpatient settings and a larger effect observed in Part B settings than is apparent with this population. We expect that other studies that include inpatient data and calculate aggregate standardized payments will generally find actual payments are higher than standardized payments, due to removal of payment add-ons, outlier payments, and geographic components (Exhibit 8). A large number of the Medicare geographic and facility-based payment differentials apply to inpatient claims.

Standardizing the payment data reduced the average PMPM payments and also greatly reduced the variability between HRRs in terms of IP payments. This setting of care has a large number of factors that are considered for the actual payments, including geographic and other types of adjustments, such 
as for various types of facilities (e.g., CAH, DSH, and teaching hospitals). For some care settings, the impact of performing the standardization was minimal. For example, the Medicare Part B payments for non-physician services (e.g., the OPTB-Other Part B setting in Exhibit 8, which included ambulance, drugs, anesthesia) were only slightly adjusted after removing the geographicallybased payment factors. Investigators will want to consider the care settings that are necessary for their study when deciding whether to invest the effort to standardize payments.

This work demonstrates that identification of areas with high or low resource use (e.g., identifying HRRs that appeared to efficiently deliver AMI care), would be very different if one did not standardize the Medicare payment data. Obtaining all of the necessary inputs for calculating standardized payments is challenging. Information regarding base rates, DRG weights, fee schedules, wage indexes, and outliers is not compiled in a central location. Since this information may change at least annually, historical information for the inputs to payment standardization should be archived in a central and publicly-accessible location.

Using completely standardized Medicare payments as a proxy for medical resources used can allow for uniformity in metrics across a wide variety of clinical topics and settings, since the units of measure are the same regardless of the illness or configuration of care studied. This sort of metric is particularly helpful when a range of services may be received over a period of time, such as hospital, skilled nursing care, home health, and physician care-when it is overly simplistic to describe resources in terms of number of hospital days or number of physician visits, for example.

Only after completely standardizing Medicare payments can we begin to ascertain whether geographic variability in payments is explained by severity of illness or regional practice patterns, in terms of the value of healthcare resources devoted to patient care. Payment information should be standardized prior to assessing whether there are differences in resource use unexplained by case mix or patient risk factors. Our study validates previous findings that there is geographic variation in resource use that is not explained by Medicare payment differentials.

\section{Disclaimer}

The content of this manuscript is solely the responsibility of the authors and does not necessarily represent the official views of the Agency for Healthcare Research and Quality, nor does it represent the official views of the Centers for Medicare \& Medicaid Services.

\section{Correspondence}

Brian O'Donnell, Ph.D., Senior Scientist, General Dynamics Information Technology, 1401-50th Street, Suite 200, West Des Moines, IA 50266, brian.odonnell@ gdit.com, Tel. 515-645-3027.

\section{Acknowledgment}

The authors would like to acknowledge the contribution of Peter Hickman, of the CMS Policy and Data Analysis Group, who was instrumental in developing the payment standardization algorithms for CMS.

\section{Financial Disclosure}

This project was supported by grant number R24HS019440 and R21HS109574 from the Agency for Healthcare Research and Quality. It also drew upon methodologies elucidated under a contract with the Centers for Medicare \& Medicaid Services (CMS), HHSM-500-2010-00001I, Order \# HHSM500-T00007.

\section{References}

Alhassani, A., Chandra, A., \& Chernew, M. E. (2012). The Sources of the SGR 'Hole.. The New England Journal of Medicine, 366, 289-291. PubMed 
Baicker, K., \& Chandra, A. (2009, October/ November). A Trillion-Dollar Geography Lesson. The variations research from Dartmouth gives us some key ingredients for raising the trillion dollars needed to pay for comprehensive coverage. Health Affairs, 28, 1448-1451. PubMed

CMS (Centers for Medicare \& Medicaid Services) (n.d.a). Medicare Hospital Quality Initiatives. Hospital Value-based Purchasing Program. Retrieved from the CMS Web site: http://www. cms.gov/HospitalQualityInits/

CMS (Centers for Medicare \& Medicaid Services) (n.d.b). Files for FY 2007 Final Rule and Correction Notice [Table 5. Acute Inpatient DRG weights]. Retrieved from the CMS Web site: http://www.cms.gov/Medicare/MedicareFee-for-Service-Payment/AcuteInpatientPPS/ Acute-Inpatient-Files-for-Download-Items/ CMS1255476.html

CMS (Centers for Medicare \& Medicaid Services) (n.d.c). Physician Fee Schedule. Retrieved from the CMS Web site: https://www.cms.gov/ PhysicianFeeSched/

CMS (Centers for Medicare \& Medicaid Services) (n.a.d). Wage index information [Acute Inpatient PPS]. Retrieved from the CMS Web site: https://www.cms.gov/AcuteInpatientPPS/03_ wageindex.asp

CMS (Centers for Medicare \& Medicaid Services) (n.d.e). QualityNet website [Measure Methodology Reports. Medicare Spending Per Beneficiary Measure]. "CMS Price Standardization" detail report. Retrieved from http://qualitynet.org/dcs/ContentServer?c=Pag e\&pagename $=$ QnetPublic\%2FPage\%2FQnetTie r4\&cid $=1228772057350$
Dartmouth Atlas. (n.d.) Downloads [Crosswalk files;ZIP codecrosswalks]. Retrieved from http:// www.dartmouthatlas.org/tools/downloads.aspx

Federal Register. (2011, November 30). Medicare and Medicaid Programs: Hospital Outpatient Prospective Payment; Ambulatory Surgical Center Payment; Hospital Value-Based Purchasing Program; Physician Self-Referral; and Patient Notification RequirementsinProviderAgreements. Retrieved from https://www.federalregister. gov/articles/2011/11/30/2011-28612/medicareand-medicaid-programs-hospital-outpatientprospective-payment-ambulatory-surgicalcenter\#p-3

Fisher, E. S., Wennberg, D. E., Stukel, T. A., Gottleib, D. J., Lucas, F. L., \& Pinder, E. L. (2003). The Implications of Regional Variations in Medicare Spending. Part 1: The Content, Quality, and Accessibility of Care. Annals of Internal Medicine, 138, 273-287. PubMed

Krumholz, H. M., Anderson, J. L., Bachelder, B. L., Fesmire, F. M., Fihn, S. D., \& Foody, J. M. ... Nallamothu, B. K. (2008) ACC/AHA 2008 Performance Measures for Adults With STElevation and Non-ST-Elevation Myocardial Infarction. A Report of the American College of Cardiology/American Heart Association Task Force on Performance Measures (Writing Committee to Develop Performance Measures for ST-Elevation and Non-STElevation Myocardial Infarction). Circulation, 118, 2596-2648. http://circ.ahajournals.org/ content/118/24/2596.full.pdf doi: http://dx.doi. org/10.1161/CIRCULATIONAHA.108.191099

Lucas, F. L., Sirovich, B. E., Gallagher, P. M., Siewers, A. E., \& Wennberg, D. E. (2010). Variation in Cardiologists' Propensity to Test and Treat. 
Is it Associated with Regional Variation in Utilization? Circulation. Cardiovascular Quality and Outcomes, 3, 253-260. PubMed

Medicare Payment Advisory Commission. (2009, December). Report to the Congress: Measuring Regional Variation in Service Use. Retrieved from the MedPAC Web site: http://www.medpac. gov/documents/Dec09_RegionalVariation_ report.pdf.

Nichols, G. A., Bell, T. J., Pedula, K. L., \& O'KeefeRosetti, M. (2010, March). Medical Care Costs Among Patients with Established Cardiovascular Disease. The American Journal of Managed Care, 16(3), e86-e93. PubMed

O'Connor, G. T., Quinton, H. B., Traven, N. D., Ramunno, L. D., Dodds, T. A., Marciniak, T. A., \& Wennberg, J. E. (1999). Geographic Variation in the Treatment of Acute Myocardial Infarction. The Cooperative Cardiovascular Project. Journal of the American Medical Association, 281, 627633. PubMed

Stukel, T. A., Fisher, E. S., Alter, D. A., Guttmann, A., Ko, D. T., Fung, K., . . Lee, D. S. (2012). Association of Hospital Spending Intensity with Mortality and Readmission Rates in Ontario Hospitals. Journal of the American Medical Association, 307(10), 1037-1045. PubMed

Wennberg, J. E., \& Cooper, M. M. (1996) “The Dartmouth Atlas of Health Care in the United States." The Trustees of Dartmouth College. http://www.dartmouthatlas.org/downloads/ atlases/96Atlas.pdf

Zuckerman, S., Waidmann, T., Berenson, R., \& Hadley, J. (2010). Clarifying Sources of Geographic Differences in Medicare Spending. The New England Journal of Medicine, 363, 54-62. PubMed 